\title{
Diagnosing primary ciliary dyskinesia: an international patient perspective
}

\author{
Laura Behan ${ }^{1,2,3}$, Audrey Dunn Galvin ${ }^{3}$, Bruna Rubbo ${ }^{1,2}$, Sarah Masefield ${ }^{4}$, \\ Fiona Copeland ${ }^{5}$, Michele Manion ${ }^{6}$, Bernhard Rindlisbacher ${ }^{7}$, Beatrice Redfern ${ }^{5}$ \\ and Jane S. Lucas ${ }^{1,2}$
}

Affiliations: ${ }^{1}$ NIHR Southampton Respiratory Biomedical Research Unit, University of Southampton Faculty of Medicine and University Hospital Southampton NHS Foundation Trust, Southampton, UK. ${ }^{2}$ Primary Ciliary Dyskinesia Centre, University Hospital Southampton NHS Foundation Trust, Southampton, UK. ${ }^{3}$ School of Applied Psychology, University College Cork, Cork, Ireland. ${ }^{4}$ European Lung Foundation, Sheffield, UK. ${ }^{5}$ Primary Ciliary Dyskinesia Family Support Group, UK. 'Primary Ciliary Dyskinesia Foundation, Minneapolis, MN, USA. ${ }^{7}$ Primary Ciliary Dyskinesia Foundation, Switzerland.

Correspondence: Laura Behan, LF100, Southampton University Hospital, Tremona Road, Southampton S016 6YD, UK. E-mail: L.Behandsoton.ac.uk

ABSTRACT Primary ciliary dyskinesia (PCD) is a rare genetic disorder characterised by progressive sinopulmonary disease, with symptoms starting soon after birth. A European Respiratory Society (ERS) Task Force aims to address disparities in diagnostics across Europe by providing evidence-based clinical practice guidelines. We aimed to identify challenges faced by patients when referred for PCD diagnostic testing.

A patient survey was developed by patient representatives and healthcare specialists to capture experience. Online versions of the survey were translated into nine languages and completed in 25 countries. Of the respondents $(n=365), 74 \%$ were PCD-positive, 5\% PCD-negative and 21\% PCDuncertain/inconclusive. We then interviewed 20 parents/patients. Transcripts were analysed thematically.

$35 \%$ of respondents visited their doctor more than 40 times with PCD-related symptoms prior to diagnostic referral. Furthermore, the most prominent theme among interviewees was a lack of PCD awareness among medical practitioners and failure to take past history into account, leading to delayed diagnosis. Patients also highlighted the need for improved reporting of results and a solution to the "inconclusive" diagnostic status.

These findings will be used to advise the ERS Task Force guidelines for diagnosing PCD, and should help stakeholders responsible for improving existing services and expanding provision for diagnosis of this rare disease.

@ERSpublications

The international PCD patients' diagnostic experience calls for earlier referral and access to specialist services http://ow.ly/lxsR300T8kO

This article has supplementary material available from erj.ersjournals.com

Received: Dec 022015 | Accepted after revision: May 262016 | First published online: Aug 042016

Conflict of interest: S. Masefield is an employee of the European Lung Foundation. Further disclosures can be found alongside this article at erj.ersjournals.com

Copyright OERS 2016. ERJ Open articles are open access and distributed under the terms of the Creative Commons Attribution Non-Commercial Licence 4.0. 


\section{Introduction}

Primary ciliary dyskinesia (PCD) is a rare heterogeneous condition. Genetic mutations cause abnormal ciliary function which is associated with abnormal ciliary ultrastructure in $70 \%$ of cases [1, 2]. PCD is characterised by impaired mucociliary clearance of upper and lower airways. Symptoms usually present soon after birth and range in severity from mild transient tachypnoea to significant neonatal respiratory failure requiring prolonged respiratory support [3-5]. Chronic and progressive chest symptoms persist throughout life, and include daily wet cough and recurrent chest infections which almost consistently lead to bronchiectasis [3]. Upper airway symptoms include rhinosinusitis and recurrent serous otitis media with hearing impairment [6]. Situs inversus is seen in approximately $40 \%$ of cases and situs ambiguous is seen in approximately $10 \%$ of cases [5].

Reported prevalence varies from 1:2000 to 1:40000, which could be due to a true variability across demographics but is likely to reflect differences in access to diagnostic facilities [7-9]. A European Respiratory Society (ERS) Task Force survey of 26 European countries found that PCD is underdiagnosed or diagnosed late, particularly in countries with low healthcare expenditure [10]. In addition to improving respiratory prognosis [11-13], early diagnosis also facilitates appropriate management of the associated upper airway disease since management is different to non-PCD-related serous otitis media and sinusitis [14]. Diagnosis also allows genetic counselling for the family.

There is no "gold standard" test to diagnose PCD and diagnostic pathways vary between/within countries [15-17]. European guidelines recommend that PCD should be confirmed in a specialist centre using appropriate diagnostic testing [18]. PCD diagnostic investigations are complex, requiring expensive infrastructure, and an experienced team of clinicians, scientists and microscopists. Due to inadequate samples or inadequate results, several samples may need to be taken until a conclusive result is achieved and the time until a conclusive result is reached can vary greatly between patients $[16,19,20]$. Various models exist to deliver diagnostic services for this rare disease, generally with a network of satellite screening centres accessing a specialist centre [20-22].

An ERS Task Force (TF-2014-04) was established in September 2014 to develop clinical practice guidelines on diagnosing or refuting the diagnosis of PCD. An important element to consider in developing practice guidelines is the patient perspective and experiences of the diagnostic process. There has been no previous international research evaluating PCD patients' experiences and their perspective on the diagnostic process. This study was therefore undertaken to address this gap and to inform the Task Force.

Over 25 years ago the authors of a pioneering study to characterise PCD concluded "all children with unexplained chronic respiratory disease, in particular those with symptoms starting in the perinatal period, should be investigated for ciliary dyskinesia" [23]. However, access of patients with these symptoms to diagnostic services remains problematic.

The present study used a mixed-methods (qualitative and quantitative) approach to more fully understand the perspectives of patients, and to identify the most prominent issues and concerns arising for patients at any stage in the diagnostic pathway.

\section{Methods}

The study combined an international web-based survey and semi-structured interviews, ensuring both breadth and depth. Ethical approval for semi-structured interviews was provided by Southampton and South West Hampshire Research Ethics Committee (A 07/Q1702/109). Audio-recorded and/or written consent was obtained from interviewees and electronic consent recorded for survey participants.

We conducted an international survey to investigate the patients' perspectives on diagnosis between June and November 2014. We designed the survey based on findings and expertise from three sources: 1) semi-structured interviews with patients from a previous study to understand factors impacting on quality of life in patients with PCD [24]; 2) consultation with patient representatives in the UK, USA and Switzerland; and 3) consultation with the European Lung Foundation (ELF). Survey topics included measures of demographics, age of diagnosis, methods of diagnosis, the patient opinions on their diagnostic experience and how diagnostic services should be delivered (box 1). To ensure it reflected wider international issues, the prototype survey was amended by ERS Task Force panel members from seven countries (UK, France, Switzerland, Germany, Italy, Ireland and Belgium). The finalised online survey (available in the online supplementary material) was translated into nine European languages (Italian, Polish, Russian, Portuguese, Czech, Dutch, German, French and Greek) using forward and back translations. We aimed to include all European countries by highlighting the study to clinicians through the ELF. Links to the survey were distributed by patient organisations, clinicians of the ERS Task Force and the ELF via newsletters, targeted emailing and social media channels. The survey was hosted by University of Southampton (www.isurvey.soton.ac.uk). 
BOX 1 Survey topics: the survey allowed participants to rate the importance of topics relating to their diagnostic experience

- The ability to meet, discuss and have their samples analysed by primary ciliary dyskinesia experts

- Repeat testing for inconclusive results

- The availability of information before testing

- The availability of information after diagnosis

- Travelling long distances for diagnostic testing

- The level of agreement on whether their condition, treatment and quality of life had improved since diagnosis

- A free text option to allow for comments was provided at the end of the survey

The survey sought the opinions of patients with PCD and parents of children with PCD. We also sought the opinions of all patients who had been considered for PCD testing irrespective of their diagnostic outcome, i.e. positive/negative/inconclusive, or by their diagnostic protocol and the tests that they received if any, i.e. nasal nitric oxide ( $\mathrm{nNO}$ ), transmission electron microscopy (TEM), high-speed video microscopy (HSVM), genetic, immunofluorescence or diagnosis based on symptoms, etc.

The survey was anonymous; however, respondents were invited to provide their email address if they wished to take part in a telephone interview. Over 80 participants provided their email address and expressed interest to be interviewed. 20 of these respondents were recruited. Efforts were made to ensure participants spoke English, and represented different age groups, diagnostic status and countries. The semi-structured interviews were conducted by the lead author (L.B.) who is trained and experienced in semi-structured/open-ended interviews and qualitative analyses, and has knowledge of PCD having developed patient-reported outcome measures for PCD (QOL-PCD [24]). Topics of discussion included: when they first heard of PCD, medical providers seen, experience with diagnostic services and any problems encountered. Telephone interviews were used to ensure a geographical representation, to provide a degree of anonymity and to relieve pressure on participants to provide the socially acceptable answer [25], i.e. discussing challenges experienced with diagnostic services and medical professionals.

\section{Data analysis}

We used simple descriptive statistics to investigate comparisons of the demographic characteristics across the range of diagnostic outcomes and between countries. Data were checked for normality and two-tailed parametric (t-test) or nonparametric (Mann-Whitney) tests were used to assess differences among mean and median values for the group as a whole and across each diagnostic outcome. Positively skewed data were transformed, where appropriate, prior to performing statistical analysis. Categorical variables were presented by frequency and percentage. Statistical analyses were performed with SPSS Statistics for Windows version 21.0 (IBM, Armonk, NY, USA).

Semi-structured interviews were recorded, transcribed, verified for accuracy and identifying information was removed. In order to maintain thoroughness, a COREQ checklist was used throughout the study [26]. Notes were maintained at all times to track decisions and help to verify the link between the original data and the findings [26]. Thematic analysis was used where patterns within data were identified using an inductive or "bottom-up" approach. By using an inductive approach, the themes identified were strongly linked to the data themselves [27]. The interview transcripts were single-coded by L.B. Themes and subthemes were discussed with the senior author J.S.L. in order to confirm the validity of the interpretations that were being developed. Data was coded using NVivo qualitative data analysis software version 10 (QSR International, Doncaster, Victoria, Australia). Recruitment ceased once saturation was reached. Themes and subthemes were presented in three stages: pre-diagnosis, diagnosis and post-diagnosis (figure 1).

\section{Results}

Study population

The survey was completed in nine European languages by 365 participants from 25 countries. The majority of respondents had been diagnosed with PCD (74.3\%), with a smaller percentage of respondents distributed across other diagnostic outcome groups. $54.5 \%$ of the respondents were parents of children and 41.6\% were adults who had been tested for PCD. $49.8 \%$ of PCD-positive respondents had situs inversus and $11.8 \%$ were born with a cardiac defect. Only one $(6 \%)$ of the PCD-negative respondents had situs inversus and $0 \%$ had a cardiac defect (table 1). Respondents were resident in Germany (21\%), the USA (18\%), France (15\%), the UK (13\%), Belgium (11\%), Denmark (5\%) and others (17\%, including Italy, 

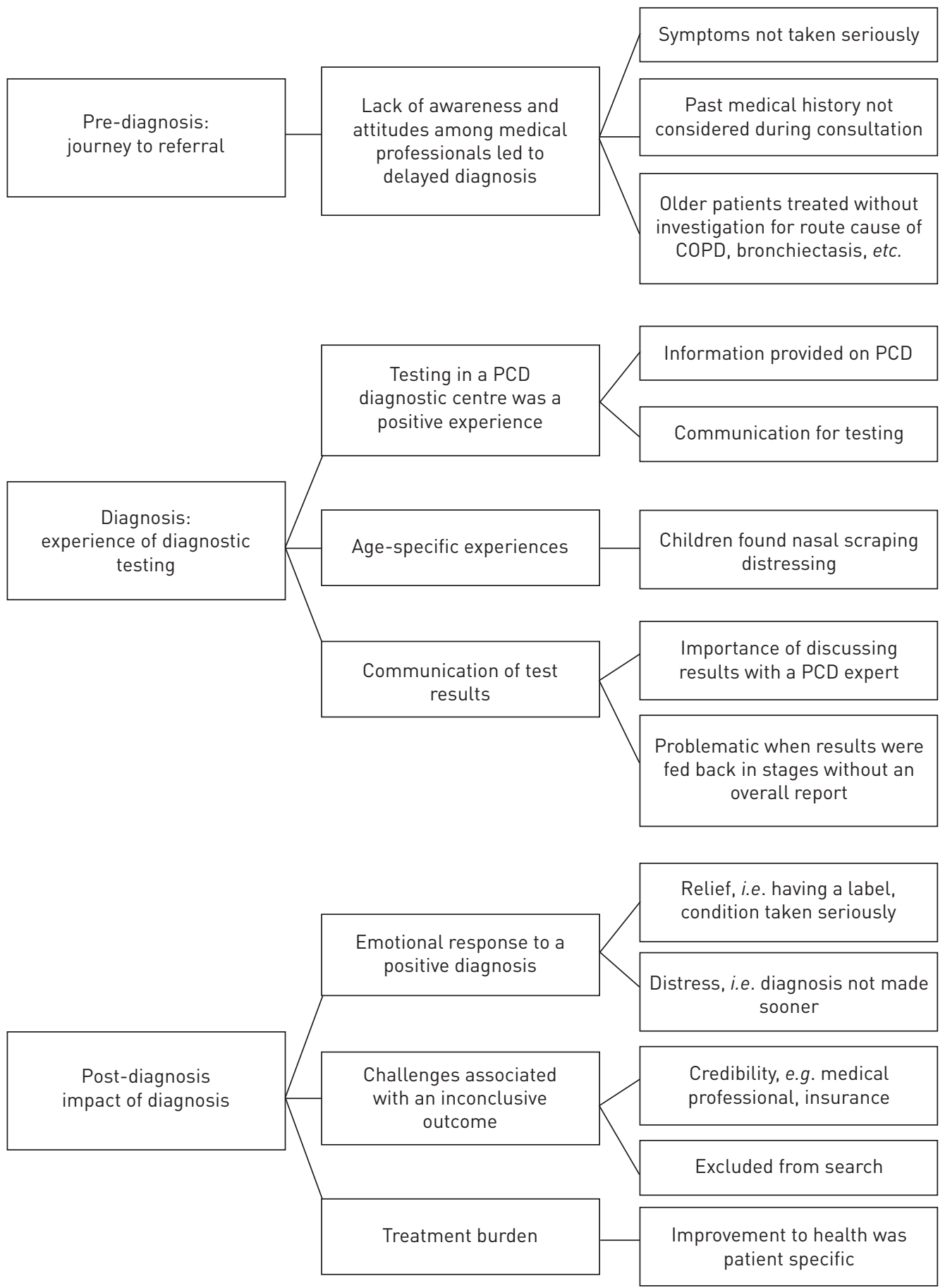

FIGURE 1 Themes and subthemes from semi-structured interviews, presented in three stages: pre-diagnosis, diagnosis and post-diagnosis. COPD: chronic obstructive pulmonary disease; PCD: primary ciliary dyskinesia.

Australia, Switzerland, Canada, Cyprus, Ireland, Greece, South Africa, Austria, Chile, Portugal, Qatar, Poland, Iran, Norway, Spain, Portugal and Algeria).

Semi-structured interviews were conducted with 20 participants from seven countries (40\% from the UK) and were diverse in age group, diagnostic testing and diagnostic outcomes (25\% were negative/inconclusive/ still waiting). $25 \%$ were parents of children aged 0-4 years, $35 \%$ were parents of children aged 5-17 years and $40 \%$ were patients tested at 18 years or older. The majority (70\%) were from English-speaking countries (online supplementary table S1). Saturation was reached after the 14th interview. 
TABLE 1 Characteristics of participants in the survey stratified by primary ciliary dyskinesia (PCD) disease status

\begin{tabular}{|c|c|c|c|c|c|}
\hline & \multirow[t]{2}{*}{ Total } & \multicolumn{4}{|c|}{ PCD disease status } \\
\hline & & Positive & Negative & Inconclusive & Other ${ }^{\#}$ \\
\hline Subjects & $365(100)$ & $271(74.3)$ & $18(4.9)$ & $29(8.0)$ & $47(12.9)$ \\
\hline Patient tested for PCD & $152(41.6)$ & $115(42.4)$ & $8(44.4)$ & $8(27.6)$ & $21(44.7)$ \\
\hline Parent of child tested for PCD & $199(54.5)$ & $152(56.1)$ & $10(55.6)$ & $21(72.4)$ & $16(34.0)$ \\
\hline Missing & $14(3.8)$ & $4(1.5)$ & & & $10(21.2)$ \\
\hline Female & $195(53.4)$ & $154(56.8)$ & 7 (38.9) & 14 (48.3) & $21(44.7)$ \\
\hline Missing & $14(3.8)$ & $3(1.1)$ & $3(16.7)$ & $1(3.5)$ & $6(12.8)$ \\
\hline \multicolumn{6}{|c|}{ Age of suspected PCD patient at time of survey years } \\
\hline$<5$ & $54(14.8)$ & $38(14.0)$ & $2(11.1)$ & $8(27.6)$ & 6 (12.8) \\
\hline $5-12$ & $88(24.1)$ & $61(22.5)$ & 7 (38.9) & $10(34.5)$ & $10(21.2)$ \\
\hline $13-17$ & $52(14.3)$ & $42(15.5)$ & $1(5.6)$ & $4(13.8)$ & $5(10.6)$ \\
\hline Missing & $12(3.29)$ & $4(1.48)$ & & & $8(17.0)$ \\
\hline Situs inversus & $157(43.0)$ & $135(49.8)$ & $1(5.6)$ & $6(20.7)$ & $15(31.9)$ \\
\hline Congenital heart defect & $41(11.2)$ & $32(11.8)$ & & $4(13.8)$ & $5(10.6)$ \\
\hline
\end{tabular}

Data are presented as $\mathrm{n}(\%) .{ }^{*}$ : other includes participants still waiting for results and those who received a false-positive result as well as missing values.

\section{Pre-diagnosis: journey to referral}

Most survey respondents had repeatedly visited medical professionals with PCD-related symptoms prior to referral; $37 \%$ of positive patients had more than 40 visits and only $3 \%$ had been referred soon after birth. $61 \%$ of PCD-negative respondents had 20 or less visits prior to referral for testing. $76.4 \%$ of respondents were older than 1 year of age when they were tested for PCD. The overall median (interquartile range) age at testing was 6 (11) years (table 2). The mean age at testing was slightly younger for those with situs inversus (mean $2.38 \pm 2.04$ versus $2.97 \pm 1.76$ for those without, $\mathrm{p}=0.016$ ) and congenital cardiac defect (mean $2.01 \pm 1.78$ versus $2.76 \pm 1.91$ for those without, $\mathrm{p}=0.036$ ). The most common reasons for diagnostic referral of interviewees was an episode(s) of pneumonia, i.e. radiography and computed tomography scan showing changes (six of 20), and self-referral following reading about PCD online (three of 20).

Interviewees attributed a delay in referral for diagnostic testing to a lack of awareness and a poor attitude among healthcare professionals (14 of 20) (figure 1). 11 of 12 parents reported that their child had respiratory distress at birth but eight of 11 were not referred until repeated visits to their physicians, sometimes over many years. Participants felt that the underlying cause of symptoms was not considered nor was past medical history taken into account. Adult participants felt they were not taken seriously, while parents reported being treated as over-cautious and over-protective. Many participants were continually treated for other common conditions, e.g. asthma and hay fever, despite treatments not having any effect. (box 2: A-D). Older participants (over 30 years at diagnosis) felt the cause of their illness was not seen as a priority for investigation due to their age with focus instead being on the treatment of chronic obstructive pulmonary disease (COPD)/bronchiectasis (box 2: A and B). 99\% of survey respondents felt that an improvement in PCD awareness amongst general practitioners and local doctors is needed to encourage early referral (figure 2).

\section{Diagnosis: experience and impact of diagnostic testing}

The most common diagnostic procedures experienced by survey respondents were nasal scraping (79\%), nNO testing (44\%) and genetic testing (37\%). $88 \%$ of survey participants rated it as important to have multiple tests if this ensured an accurate result and to see a PCD expert even if it means travelling long distances (figure 2). This was echoed overall in the interviews (box 3: A-C); however, some age-specific variances emerged with parents describing nasal scraping as a moderately distressing experience. This distress was heightened if their child needed a repeat brushing (box 3: B and C). Participants reported that testing was carried out in an efficient and empathetic manner with the appropriate amount of information provided once they reached specialist services (eight of 20) (box 3: D and E). 
TABLE 2 Age at testing, time taken to receive test results and number of visits to doctor before referral stratified by primary ciliary dyskinesia (PCD) status for survey participants

\begin{tabular}{|c|c|c|c|c|c|}
\hline & \multirow[t]{2}{*}{ Total } & \multicolumn{4}{|c|}{ PCD disease status } \\
\hline & & Positive & Negative & Inconclusive & Other ${ }^{\#}$ \\
\hline Subjects & $365(100)$ & $271(74.3)$ & $18(4.9)$ & $29(8.0)$ & 47 (12.9) \\
\hline$<1$ & $42(11.5)$ & $30(11.1)$ & & $4(13.8)$ & $8(17.0)$ \\
\hline$\geqslant 1$ & $279(76.4)$ & $229(84.5)$ & $14(77.8)$ & $20(69.0)$ & $16(34.0)$ \\
\hline Missing & $44(12.1)$ & $12(4.4)$ & $4(22.2)$ & $5(17.2)$ & 23 (48.9) \\
\hline $1-10$ & $64(17.5)$ & $46(17.0)$ & 6 (33.3) & 5 (17.3) & 7 (14.2) \\
\hline $11-20$ & $56(15.3)$ & $45(16.6)$ & $5(27.8)$ & $3(10.3)$ & $3(6.4)$ \\
\hline $21-40$ & $68(18.6)$ & 52 (19.2) & $4(22.2)$ & $8(27.6)$ & $4(8.5)$ \\
\hline$>40$ & 123 (33.7) & $101(37.3)$ & $1(5.6)$ & $8(27.6)$ & $13(27.7)$ \\
\hline Other & $22(6.03)$ & $13(4.8)$ & $1(5.6)$ & $3(10.3)$ & $5(10.6)$ \\
\hline \multicolumn{6}{|l|}{ Time to diagnosis after testing for PCD } \\
\hline$>1$ year & $28(7.7)$ & $24(8.9)$ & $1(5.6)$ & $2(6.9)$ & $1(2.1)$ \\
\hline Still waiting & $28(7.7)$ & & & $15(51.7)$ & 13 (27.7) \\
\hline Not sure & $36(9.9)$ & $31(11.4)$ & & $2(6.9)$ & $3(6.4)$ \\
\hline Missing & $20(5.5)$ & $3(1.1)$ & & $1(3.5)$ & $16(34.0)$ \\
\hline \multicolumn{6}{|l|}{ Diagnostic procedures } \\
\hline Nasal nitric oxide & $157(43.0)$ & $110(40.6)$ & $14(77.8)$ & $17(58.6)$ & $16(34.0)$ \\
\hline Nasal scraping & $283(77.5)$ & $221(81.6)$ & 16 [88.9] & $28(96.6)$ & 18 (38.3) \\
\hline Bronchoscopy & $134(36.7)$ & $102(37.6)$ & 6 (33.3) & $12(41.4)$ & $14(29.8)$ \\
\hline Genetics & $134(36.7)$ & $105(38.8)$ & $1(5.6)$ & $13(44.8)$ & $15(31.9)$ \\
\hline Saccharine test & $19(5.2)$ & $14(5.2)$ & $1(5.6)$ & $2(6.9)$ & $2(4.3)$ \\
\hline Nuclear medicine scan (e.g. MRI and CT scans) & $25(6.9)$ & $19(7.0)$ & $2(11.1)$ & $1(3.5)$ & $3(6.4)$ \\
\hline Radiography or CT scan alone & $8(2.2)$ & $2(0.7)$ & & & 6 (12.8) \\
\hline
\end{tabular}

Data are presented as $\mathrm{n}(\%)$. MRI: magnetic resonance imaging; CT: computed tomography. ${ }^{\#}$ : other includes participants still waiting for results and those who received a false-positive result as well as missing value; ${ }^{\text {I }}$ : median age (interquartile range) 6 (11) years (n=226).

Diagnosis: awaiting and receiving results

A total of $67 \%$ of survey respondents received their result within a 6 months period and $12 \%$ reported that they received confirmatory results within 1 week of testing (table 2). Opinions varied on what was deemed to be a "long time" to wait for results (box 4: A and B). $94 \%$ of survey participants rated having samples analysed by a PCD expert as important or very important and $97 \%$ rated having the opportunity to discuss results with a PCD expert as important or very important (figure 2).

Participants referred to encountering problems when results for tests such as TEM and genetics were received much later than others, e.g. HSVM and nNO test. Explanations of what the results mean in combination were incorrectly relayed back to the patients. To overcome this, participants recommended that it would be helpful if they could receive an overall report explaining what the results mean in combination and to have the opportunity to discuss this with a PCD expert (box 4: D and E).

\section{Post-diagnosis: impact of diagnosis}

Participants who experienced an inconclusive diagnostic result discussed the practical constraints of such an outcome, e.g. insurance constraints and not being included in research (box 5: A and B). The emotional impact of a positive diagnosis was described by many as leading to a profound sense of validation that their symptoms were down to a specific named condition (box 5: C and D). Participants also reported feeling some distress that their/their child's diagnosis process took such a long time and felt that an earlier diagnosis could have made a significant difference to the state of their current and future health; relief was expressed once they had a label and could pursue getting the correct treatment (box 5: E and F). Relief was also described when a negative diagnosis was received (box 5: G). 


\section{BOX 2 Subthemes and participant quotes for pre-diagnostic testing: journey to referral}

\section{Past medical history was not considered}

A It was a wait, but it was more the fact that no-one was looking at the history ... she'd been in neonatal for eleven days when she was born, she was full-term ... she couldn't get her sats up ... how many times I'd been in, you know and no one put two and two together. [Participant 010, parent, positive PCD]

\section{Symptoms not taken seriously: parents treated as fussy/over protective}

B ... and all this time you know ... time's going on ... I don't think that I was listened to as a mum, it was a bit like, I was you know maybe of over worrying, things like that. [Participant 004, parent, positive $P C D]$

C I think the lateness in the diagnosis has caused the bronchiectasis and ... other problems and I think that could have been prevented if someone would have taken me a bit more seriously. [Participant 010, parent, positive $P C D$ ]

\section{Mistreated for other illnesses}

D ... he treated her for asthma, but the picture never made sense, and she didn't look like an asthmatic child. [Participant 015, parent, positive PCD]

Older patient treated without investigation for route cause

E There seems to be, among pneumologists, that they think in adult people or at least people who are more than forty years old, it doesn't matter anymore which is the reason for this COPD, and they don't even think about testing it. [Participant 011, adult, positive PCD]

PCD: primary ciliary dyskinesia.

\section{Post-diagnosis: follow-up care and support available}

Participants expressed a sense of being overwhelmed by the prescription of medication, treatments and clinic appointments following a positive diagnosis, particularly when newly diagnosed. The benefits of a diagnosis to the reported current state of health varied among survey participants with $21 \%$ reporting no improvement to health since diagnosis (figure 2). A significant difference was found between those who were diagnosed in childhood (0-12 years) and those who were diagnosed in adolescence/adulthood (over 13 years $)$ on the level of agreement that health has improved since diagnosis $(\mathrm{p}=0.041)$. Similar variability was found among the interviewees with some participants feeling that their/their child's health had benefited greatly since starting treatments (box 5: A-C) and others reporting no major change since diagnosis despite adhering to the prescribed treatments (box 5: D).

In the survey, $91 \%$ of respondents strongly agreed/agreed that it is important to have a patient organisation in each country (figure 2). The role of PCD patient support groups following referral was further elaborated in the interviews as important in terms of meeting other families affected by PCD and having an opportunity to share experiences (box 6: E and F). Participants in countries without a PCD support group spoke of the need for support services enabling discussions about experiences specific to their country's health system and in their native language (box 6: G-I).

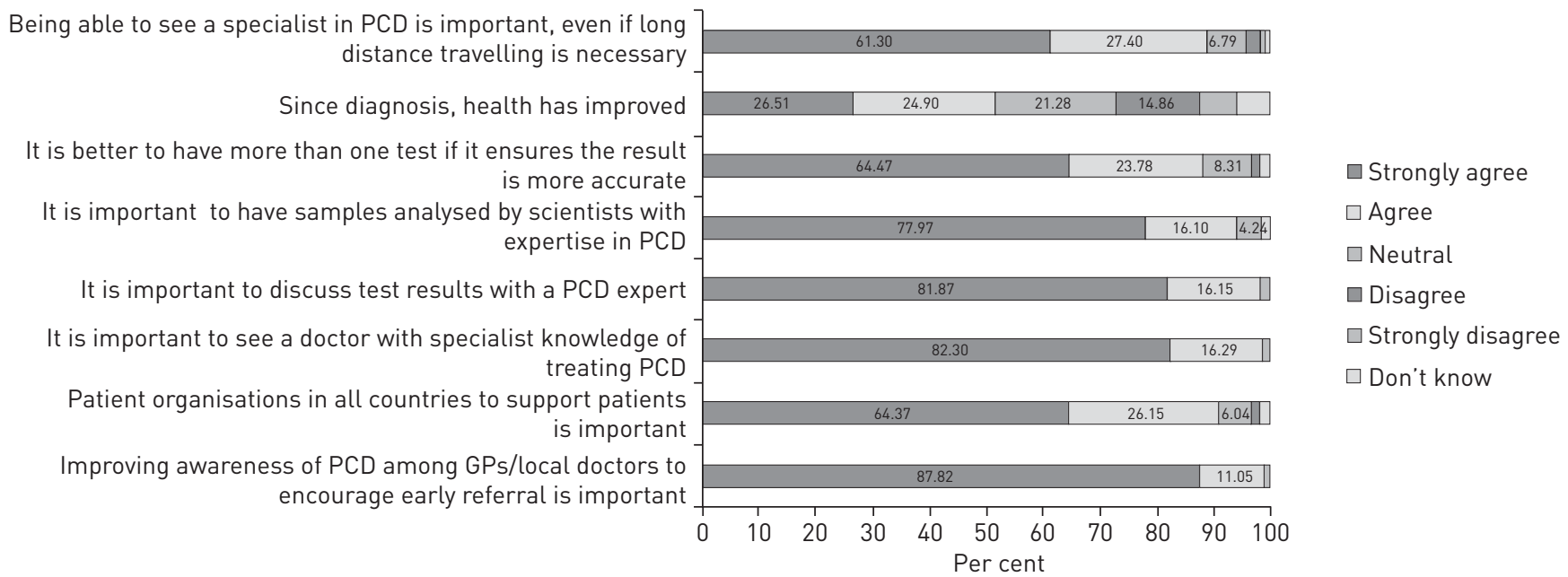

FIGURE 2 Survey findings on patient opinion of diagnostic effect on health and the importance of expert service provision. PCD: primary ciliary dyskinesia; GP: general practitioner. 


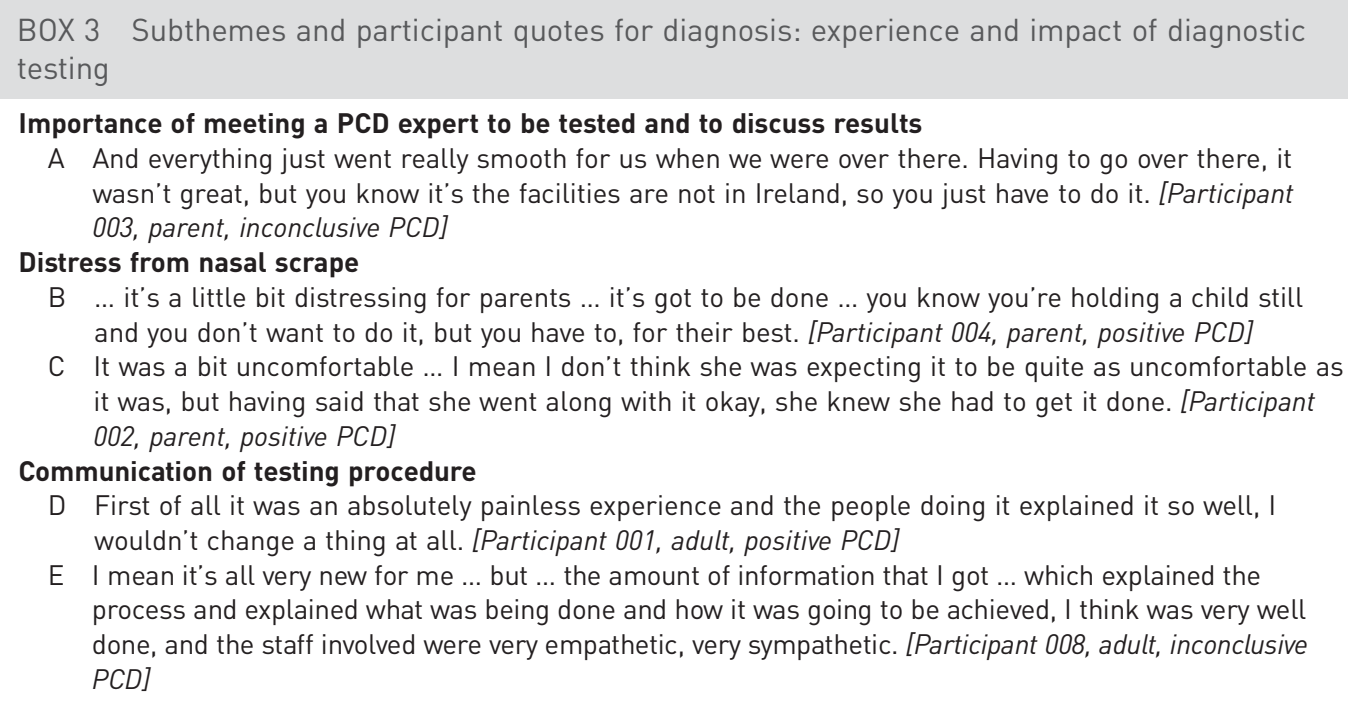

PCD: primary ciliary dyskinesia.

\section{Discussion}

This study represents findings from an international survey and in-depth semi-structured interviews specifically designed to understand the experiences, concerns and needs of patients requiring diagnostic testing for PCD. It describes the experiences and perspectives of 365 adult patients and parents from 25 European Union (EU) and non-EU countries, 20 of whom were interviewed to obtain an in-depth insight to their experiences and opinions of being tested for PCD.

When discussing the journey to referral, the overarching theme was the lack of PCD awareness by medical professionals thus leading to a delayed diagnosis. This was also reflected in the survey, with $34 \%$ of the total respondents reporting that they visited their doctor on more than 40 occasions for PCD-related symptoms before PCD was considered a possible diagnosis. There is a clear need to reiterate to nonexpert clinicians the guidelines [23] published a quarter of a century ago, which stated that individuals with chronic upper and lower airway symptoms should be investigated for PCD. Previous research has found a sense of isolation and mistrust in medical care among PCD patients which is heightened by a lack of PCD awareness by the patient's general practitioner [28]. Participants were concerned that delayed diagnosis had adversely affected their health. All adult interviewees were diagnosed late in life (over 30 years of age) and felt that their current state of health might have been better had they been diagnosed earlier. This

BOX 4 Subthemes and participant quotes for diagnosis: awaiting and receiving results

\section{Long-time taken to receive results}

A I had to ring up months after, chasing up the results ... we should have got the results sooner than what we did ... like the testing was done in the January and probably should have got the results probably March, April. Ended up being like June, July, because I had to actually chase up myself to get the results. [Participant 019, parent, positive PCD]

B No, definitely a month is a long time to wait. To know what's wrong with your child or significant other or something, but it takes as long as it takes; I have gotten used to that. [Participant 017, parent, positive $P C D]$

\section{Experience of communication of diagnostic results}

C And I got the results, let's say in drops, maybe one year later I got the information about the electron microscopy was or maybe it was eighteen months later. [Participant 011, adult, positive PCD]

D I got the information from my doctor that my son ... probably has PCD ... the cilia didn't move ... about already 1 month later ... the report that the electron microscopy was normal, and the report from the electron microscopy said we cannot prove primary ciliary dyskinesia based on this. The doctor told me, "well then it's not primary ciliary dyskinesia". [Survey participant, open text response]

E Before they did any testing we had a good discussion about what it was and what it meant, and then when we got the results they explained what it was that wasn't working ... It's usually better if somebody talks to you rather than it being written down. [Participant 010, parent, positive PCD]

PCD: primary ciliary dyskinesia. 


\section{BOX 5 Subthemes and participant quotes for post-diagnosis: impact of diagnosis}

\section{Impact of an inconclusive diagnostic result}

A I have suffered from serious upper and lower respiratory infections all my life. I have an "inconclusive" status re: diagnosis of PCD ... there must be a category of PCD that includes a variant of PCD as a diagnostic category. This is crucial for people who have insurance challenges. [Survey participant, open text response]

B I hate the probable PCD diagnosis. Four years ago my three kids were given that. All classic symptoms with bronchiectasis and low nasal nNO. But cilia appeared normal. It infuriates me they can't be included in research. [Survey participant, open text response]

Emotional response to positive diagnosis: feeling of validation

C Like to have the confirmed diagnosis ... well I am just really excited they were finally able to prove it's not in my head. You know like I didn't make it up. [Participant 016, adult, positive PCD]

D I actually felt like shouting Alleluia once she was diagnosed, because it was just years and years of worry and thinking what's going on and why, and I just, you kind of doubt yourself as a mum. [Participant 010, parent, positive PCD]

Emotional response to positive diagnosis: relief but a sense of sadness that diagnosis was not sooner

$E$ You know that's kind of like oh wow, finally I'm no longer a book-case. But then I have all this damage that you know at least, you know at least I have a diagnosis, twenty years later, but it's a little late. [Participant 009, adult, positive PCD]

F Well it was a certain relief, first of all in that you know we can explain now ... we can help her in the way that she needs. So really it was just a huge kind of relief really, just knowing that we have an explanation now. Along with you know a bit of sadness that we didn't catch onto it earlier. [Participant 002, parent, positive $P C D]$

\section{Impact of a negative diagnosis: relief}

G I was very relieved of course. I didn't know anything at all about it until I was told by the consultant I was going to be sent for the test. And then I received some documentation in advance with the appointment. And I also read a little bit about it online ... I was quite concerned, because one of the things I heard was that it could affect your hearing and you can go deaf ... I was quite anxious, so the length of time being referred and actually having the tests, I was very worried. [Participant 007, adult, negative $P C D]$

PCD: primary ciliary dyskinesia.

finding supports previous research that found that delayed PCD diagnosis led to poorer subsequent quality of life [29]. To prevent a delayed diagnosis, participants felt that medical professionals need be to be better informed about PCD, its signs and symptoms, and relevance of past medical history. It is hoped that increased availability of screening tools such as nNO [30] and clinical prediction rules (e.g. PICADAR) $[31,32]$ will increase identification of appropriate patients for diagnostic testing and reduce delays. However, if the screening tools are used in patient populations with a low likelihood of PCD there is a risk that excessive numbers of patients will be referred to specialist services, resulting in unnecessary testing for low-risk patients whilst overwhelming diagnostic centres [33].

When receiving a diagnosis, patient and parent interviewees who visited specialised diagnostic centres were satisfied with their experience. The implications of normal electron microscopy wrongly being interpreted as negative diagnosis [17] by non-PCD experts was highlighted. Respondents felt that being able to discuss their test results with a PCD expert as well as receiving a written report of all test results was important, and contributed to a sense of certainty and assurance.

Due to the importance of societal, health and cultural differences between countries, we aimed to recruit participants from diverse geographical regions. We first examined the results from all participants and then analysed countries separately. A quantitative comparison of countries grouped by general government expenditure on health (the sum of outlays for health maintenance, restoration or enhancement paid for by government entities) or grouped by countries with and without specialist diagnostic services was prevented due to the limited numbers of respondents from some areas. We have, however, included the analyses from the UK, Germany and the USA to show comparisons with differing approaches to delivery of diagnostic services and availability of tests. The percentage of respondents who had 40 or more visits to their doctor with symptoms related to PCD before being referred for testing was significantly higher in the USA (44\%) compared with the UK (24\%) and Germany (35\%) (online supplementary table S2). The interviews also revealed country-specific variances. Participants from non-English-speaking countries strongly advocated that support groups be set up in their country and for information to be available online in all languages. The inconclusive PCD diagnosis also had country-specific implications, with participants from countries including the USA requiring a definitive diagnosis in order to access insurance 
BOX 6 Subthemes and participant quotes for post-diagnosis: follow-up care and support available

\section{Treatment burden and effect on condition}

A It was a bit of a shock ... I was probably in my mid-thirties then, to suddenly be told, right, you've got to do twenty minutes of physio twice a day, you've got to take this blue puffer, and the brown puffer ... as soon as you get a chest infection you've got to take really strong antibiotics, I rebelled against that, just because it was too much all at once. [Participant 006, adult, positive PCD]

$B$ For me it made a huge difference in my treatment when I finally knew the diagnosis. And I am certain that I wouldn't be as well as I still am when I would have continued in the way as I did before it. [Participant 011, adult, positive PCD]

C She was sick every month. Once we had a diagnosis ... she gets sick, but not as severe as ... before. Definitely milder ... you know we have a treatment plan and even when she starts to get sick; those medications are changed so we tend to catch that right away rather than after that. [Participant 015, parent, positive $P C D]$

D It's strange, since being diagnosed we are now bombarded with a very heavy burden of care, she uses the nebulisers three times a day, and she has physio twice a day ... and yet she's not clinically, she's no different to before she was diagnosed. [Participant 004, parent, positive PCD]

Support from PCD patient organisations

E Well we go, after the diagnosis ... through the PCD Foundation, and that was a huge support. Also there is like chat groups that we go on. We've gone to family days. So we've met quite a few other families that also have PCD ... yeah, it's a huge, huge help really. Sometimes it's even just finding out what they did in certain situations. So things you can bring up to your doctor, so that's helpful as well. [Participant 015, parent, positive PCD]

F I got connected with the PCD group online ... without the people in this group ... I would be terribly lost ... they've been my life support. [Participant 009, adult patient, positive PCD]

Need for PCD patient organisations in each country

G In Spain, we would like ... a Patients Association ..., in the UK there will be one of these, you know this association, but in Spain we don't know anything about the illness and the patients. [Participant 013, parent, positive $P C D]$

$\mathrm{H}$ There isn't any in Ireland ... I don't know anybody else that has it, and if I did know somebody that would be handy ... like you could see ... what does the future hold for us in a few years' time, really we're only going day-by-day, and I know every case is different, but it would be nice to kind of know other issues some people have experienced. And the mistakes I made, maybe you could try this, which might help. [Participant 003, parent, inconclusive PCD]

I I think it's difficult. There is no information in Bulgarian for example ... and I would speak English so I can get a lot of information from the internet, but if you are a Bulgarian and you do not speak English, it's very, very hard for you to get that information. [Participant 012, adult patient, probable PCD]

PCD: primary ciliary dyskinesia.

for treatments. A resolution to the problems associated with an inconclusive result or a "probable" PCD diagnosis was called for.

\section{Strengths and limitations}

This is the first international study of the perspective of patients referred for PCD diagnostic testing. Questions for the survey involved the contribution of an international panel of PCD experts and patient representatives. However, our study does have limitations: response varies from country to country, with the main percentage of respondents from countries with established diagnostic centres, i.e. the UK, USA, Germany and France.

The survey also aimed to include patients who were referred and still waiting for results, who were negative for PCD, or who have been found to be inconclusive for PCD and are still going through testing; however, $74.3 \%$ of survey respondents were PCD-positive, with PCD-negative representing only $4.9 \%$, inconclusive $8 \%$ and still waiting $3 \%$. It is especially low considering between $11.5 \%$ and $18.6 \%$ of referrals are eventually diagnosed with PCD [17, 31-33]. It is perhaps not surprising that patients with ongoing interest in the disease (PCD-positive) were more likely to respond to the survey. This limits our capacity to assess the perspective of the population with an inconclusive or negative outcome.

Although participants were asked about the tests that had been performed, their results cannot be verified and there is therefore diagnostic uncertainty. In addition, a number of participants' diagnoses were based on tests that are not considered robust, e.g. the saccharine test. It is therefore likely that some patients may have been diagnosed who do not have PCD and vice versa. Since we were interested in the experiences and outcomes of patients from diverse clinical settings it was important to include groups where diagnostic 
status was uncertain. This inherently means that allocation of patients to diagnostic outcome groups was defined by the participants' understanding rather than the diagnosis that might be provided by a highly specialist centre; this is at the same time a strength and a weakness of the study.

\section{Implications and conclusion}

This study concludes that there are a number of recommendations for the improvement of PCD diagnosis from the patient's perspective and that are needed across Europe and internationally. 1) Samples should be analysed by PCD experts. 2) Results should be delivered by a PCD expert. Patients should have the opportunity to discuss their results with a PCD expert and to ask questions. 3) Repeat testing should be completed if needed, to ensure an accurate result. 4) Measures must be introduced to prevent late diagnosis such as better knowledge of PCD by medical practitioners, including relevance of past medical history. 4) A resolution to the "inconclusive" diagnosis result status. 5) Establishment of a patient support group in each country. 6) Availability of online translated information on PCD in all European languages

This is the first international study evaluating PCD patients' experiences and their perspective on the diagnostic process. The findings from this study will be used to advise the ERS Task Force (TF-2014-04) as they develop clinical practice guidelines on diagnosing or refuting the diagnosis of PCD. We anticipate that the results will inform stakeholders with responsibility for improving existing diagnostic provision and for expanding services for this rare disease. It should feed into the new European Reference Networks (ERNs) for rare diseases, particularly the PCD ERN.

\section{Acknowledgements}

This work was conducted to inform the development of diagnostic guidelines for PCD by ERS Task Force TF-2014-04. The authors of this manuscript are participants in BEAT-PCD (COST Action 1407). We would like to thank all those who were involved in the development, translation and international distribution of this survey, in particular: Mieke Boon (University Hospital of Leuven, Leuven, Belgium), Nicolas Schwerk (Hannover Medical School, Hannover, Germany), Ernst Ebber (Medical University of Graz, Graz, Austria), Folke Brinkman (Hannover Medical School, Hannover, Germany), Jean Francois Papon (Université Paris-Est Créteil, Créteil, France), Katarzina Wolnowska (Polish Academy of Sciences, Warsaw, Poland), Zuzanna Bukowy-Bieryło (Polish Academy of Sciences, Warsaw, Poland), Susanna Lopez (Universidade Nova de Lisboa, Lisboa, Portugal), Francesca Santamaria (Federico II University, Naples, Italy), Claudia Kuenhi (University of Bern, Bern, Switzerland), Myroflora Goutaki (University of Bern, Bern, Switzerland), Heymut Omran (University Children's Hospital Muenster, Muenster, Germany), Panagiotis Kouis (Cyprus University of Technology, Limassol, Cyprus), Panagiotis Yiallouros (Cyprus University of Technology, Limassol, Cyprus), Kostas Priftis (Attikon General Hospital, Athens, Greece), Manos Papadakis (Southampton General Hospital, Southampton, UK), Jamila McRobb (University Hospital Southampton, Southampton, UK) and David A. Johnston (University of Southampton, Southampton, UK). We would also like to thank the patients and patient organisation representatives who informed the development of the survey, and the survey respondents and interview participants for sharing their experiences and perspectives.

\section{References}

$1 \quad$ Afzelius B. A human syndrome caused by immotile cilia. Science 1976; 193: 317-319.

2 Chilvers MA, Rutman A, O'Callaghan C. Ciliary beat pattern is associated with specific ultrastructural defects in primary ciliary dyskinesia. J Allergy Clin Immunol 2003; 112: 518-524.

3 Noone PG, Leigh MW, Sannuti A, et al. Primary ciliary dyskinesia: diagnostic and phenotypic features. Am J Respir Crit Care Med 2004; 169: 459-467.

4 Coren M, Meeks M, Morrison I, et al. Primary ciliary dyskinesia: age at diagnosis and symptom history. Acta Paediatr 2003; 91: 667-669.

5 Mullowney T, Manson D, Kim R, et al. Primary ciliary dyskinesia and neonatal respiratory distress. Pediatrics 2014; 134: 1160-1166.

6 Pedersen M, Mygind N. Rhinitis, sinusitis and otitis media in Kartagener's syndrome (primary ciliary dyskinesia). Clin Otolaryngol Allied Sci 1982; 7: 373-380.

7 Kuehni CE, Frischer T, Strippoli MP, et al. Factors influencing age at diagnosis of primary ciliary dyskinesia in European children. Eur Respir J 2010; 36: 1248-1258.

8 Lucas JS, Walker WT, Kuehni CE, et al. Primary ciliary dyskinesia. In: Courdier J-F, ed. Orphan Lung Diseases. ERS Monogr 2011; 54: 201-217.

9 O'Callaghan C, Chetcuti P, Moya E. High prevalence of primary ciliary dyskinesia in a British Asian population. Arch Dis Child 2010; 95: 51-52.

10 Kuehni CE, Frischer T, Strippoli MP, et al. Factors influencing age at diagnosis of primary ciliary dyskinesia in European children. Eur Respir J 2010; 36: 1248-1258.

11 Ellerman A, Bisgaard H. Longitudinal study of lung function in a cohort of primary ciliary dyskinesia. Eur Respir J 1997; 10: 2376-2379.

12 Hellinckx J, Demedts M, De Boeck K. Primary ciliary dyskinesia: evolution of pulmonary function. Eur J Pediatr 1998; 157: 422-426.

13 Marthin JK, Petersen N, Skovgaard LT, et al. Lung function in patients with primary ciliary dyskinesia: a cross-sectional and 3-decade longitudinal study. Am J Respir Crit Care Med 2010; 181: 1262-1268.

14 Lucas JS, Burgess A, Mitchison HM, et al. Diagnosis and management of primary ciliary dyskinesia. Arch Dis Child 2014; 99: 850-856.

15 Lucas JS, Paff T, Goggin P, et al. Diagnostic methods in primary ciliary dyskinesia. Paediatr Respir Rev 2016; 18: 8-17. 
16 Lucas JS, Leigh MW. Diagnosis of primary ciliary dyskinesia: searching for a gold standard. Eur Respir J 2014; 44: $1418-1422$.

17 Jackson CBL, Collins SA, Goggin PM, et al. Accuracy of diagnostic testing in primary ciliary dyskinesia. Eur Respir J 2016; 47: 837-848.

18 Barbato A, Frischer T, Kuehni CE, et al. Primary ciliary dyskinesia: a consensus statement on diagnostic and treatment approaches in children. Eur Respir J 2009; 34: 1264-1276.

19 O'Callaghan C, Chilvers M, Hogg C, et al. Diagnosing primary ciliary dyskinesia. Thorax 2007; 62: 656-657.

20 Knowles MR, Daniels LA, Davis SD, et al. Primary ciliary dyskinesia. Recent advances in diagnostics, genetics, and characterization of clinical disease. Am J Respir Crit Care Med 2013; 188: 913-922.

21 Leigh MW, Hazucha MJ, Chawla KK, et al. Standardizing nasal nitric oxide measurement as a test for primary ciliary dyskinesia. Ann Am Thorac Soc 2013; 10: 574-581.

22 Lucas JS, Burgess A, Mitchison HM, et al. Diagnosis and management of primary ciliary dyskinesia. Arch Dis Child 2014; 99: 850-856.

23 Buchdahl RM, Reiser J, Ingram D, et al. Ciliary abnormalities in respiratory disease. Arch Dis Child 1988; 63: 238-243.

24 Lucas JS, Behan L, Dunn Galvin A, et al. A quality-of-life measure for adults with primary ciliary dyskinesia: QOL-PCD. Eur Respir J 2015; 46: 375-383.

25 Fenig S, Levav I, Kohn R, et al. Telephone vs face-to-face interviewing in a community psychiatric survey. Am J Public Health 1993; 83: 896-898.

26 Tong A, Sainsbury P, Craig J. Consolidated criteria for reporting qualitative research (COREQ): a 32-item checklist for interviews and focus groups. Int J Qual Health Care 2007; 19: 349-357.

27 Braun V, Clarke V. Using thematic analysis in psychology. Qual Res Psychol 2006; 3: 77-101.

28 Whalley S, McManus IC. Living with primary ciliary dyskinesia: a prospective qualitative study of knowledge sharing, symptom concealment, embarrassment, mistrust, and stigma. BMC Pulm Med 2006; 6: 25.

29 Pifferi M, Bush A, Di Cicco M, et al. Health-related quality of life and unmet needs in patients with primary ciliary dyskinesia. Eur Respir J 2010; 35: 787-794

30 Collins SA, Gove K, Walker W, et al. Nasal nitric oxide screening for primary ciliary dyskinesia: systematic review and meta-analysis. Eur Respir J 2014; 44: 1589-1599.

31 Behan L, Dimitrov BD, Kuehni CE, et al. PICADAR: a diagnostic predictive tool for primary ciliary dyskinesia. Eur Respir J 2016; 47: 1103-1112.

32 Leigh MW, Ferkol TW, Davis SD, et al. Clinical features and associated likelihood of primary ciliary dyskinesia in children and adolescents. Ann Am Thorac Soc 2016 [in press; DOI: 10.1513/AnnalsATS.201511-748OC].

33 Collins SA, Behan L, Harris A, et al. The dangers of widespread nitric oxide screening for primary ciliary dyskinesia. Thorax 2016; 71: 560-561. 